\title{
14
}

\section{MOSQUITO CONTROL}

\section{Success, failure and expectations in the context of arbovirus expansion and emergence}

\author{
Isabelle Dusfour and Sarah C. Chaney
}

Mosquitoes are considered humanity's most dangerous animal due to their capability to transmit a large number of deadly viruses and parasites, causing millions of illnesses and deaths annually, along with enormous economic loss (WHO 2020, Bradshaw et al. 2016). Among the 3,500 known mosquito species, however, only a few are vectors of these pathogens.

A vector is a mosquito that is able to pick up, amplify and transmit a pathogen from one vertebrate to another through blood feeding (Marcondes 2019). Only female mosquitoes are hematophagous (blood-feeders) and are therefore responsible for all mosquito-borne disease transmission. Yet not all female mosquitoes can transmit pathogens and some are better at it than others: the ability of any given mosquito to transmit disease from one vertebrate to another depends on its behaviour, how it fits into the ecosystem of the human-built and the natural world, and its internal biology. Each of these factors can be targeted with vector control strategies to interrupt the transmission of disease.

One way of reducing mosquito populations is to alter environmental factors necessary for mosquito breeding or to apply chemicals that target flying adults (adulticiding) or immature aquatic larval stages (larviciding). However, as we will see as we explore successes and failures of mosquito control, many chemical tools seem to have reached the end of their effectiveness as a stand-alone strategy. Despite decades of chemical control efforts over the past 50 years, the world has faced the intensification of dengue outbreaks, the re-emergence of yellow fever, the spread of chikungunya and Zika and the emergence of zoonotic diseases accompanied by the geographical expansion of major vectors (WilderSmith et al. 2017). Vector control departments are now faced with a challenge to expand beyond immediate prevention of human disease towards a global approach that encompasses the biology, behaviour and biodiversity of mosquito species, their ecology and what makes them effective or ineffective vectors-in 
order to formulate a realistic, multi-faceted, environmentally friendly and efficient mosquito control strategy.

This chapter reviews what has been done so far to control Aedes aegypti, current challenges raised by the expansion of Aedes albopictus and growing threats of zoonotic viruses, and what innovations are under development to reduce mosquito-borne transmission of viruses. French overseas territories and the Americas are the focus here due to the special challenges they are facing to control mosquito vectors.

\section{Controlling Aedes species in urban areas: Aedes aegypti and Aedes albopictus}

Aedes aegypti and Aedes albopictus are the main urban vectors of arboviruses, the arthropod-borne viruses of yellow fever, dengue, chikungunya and Zika that threaten more than 3 billion people living in Aedes-infected areas worldwide (Wilder-Smith et al. 2017). Aedes species are optimally adapted for transmitting viruses from human to human: they can carry multiple arboviruses, are anthropophilic (prefer humans for blood feeding), bite during the day and feed multiple times, fly only short distances and prefer to breed in small human-made containers. Additionally, Aedes eggs are resistant to desiccation, giving them the advantage of spreading their offspring to new territories worldwide through human travel and commercial trade (Kraemer et al. 2019, Marcondes 2019).

Even though the two dominant Aedes vector species look very similar, their biology, ecology, behaviour and history of colonization reveal important differences. Ae. aegypti originated from forests of the western part of Africa and began spreading around the world through the transatlantic slave trade in the sixteenth century (Powell et al. 2018). This forest-dwelling species adapted to urban areas by becoming more anthropophilic and breeding specifically in human-made containers such as cisterns and buckets. This urban mosquito is closely tied to human habitation in all steps of its life cycle and is found less abundantly in rural areas and rarely in natural breeding sites. Ae. aegypti has colonized urban areas of subtropical and tropical regions around the world and is the main vector of the yellow fever virus, along with dengue viruses, and more recently chikungunya and Zika viruses.

Aedes albopictus, the Asian tiger mosquito, originates from forests in Asia and has become the world's most invasive mosquito species, colonizing all areas of the planet over the last 30 years within its preferred temperature range. Even though its ecological niche seems similar to that of Ae. aegypti, this species tends to be more rural, develops in a larger variety of natural and human-made breeding sites and adapts to a wide range of temperatures due to its capacity to lay cold-resistant eggs that can survive winter temperatures during its diapause stage (Paupy et al. 2009). Ae. albopictus is also less selective about hosts and can be found feeding on animals as well as humans. It does not transmit dengue and yellow fever quite as efficiently as Ae. aegypti and has until recently been considered more of 
a nuisance than a public health concern. However, Ae. albopictus revealed its true colours during the 2006-2007 chikungunya outbreak that hit the Indian Ocean Islands, Central Africa, India and Italy. Ae. Albopictus' growing distribution to more temperate latitudes and its ability to transmit about 26 different arboviruses between human and animal hosts makes it a growing threat in temperate regions (Paupy et al. 2009).

In some areas, the ranges of these two species overlap, sharing resources, often laying eggs in the same breeding sites and intermingling in ways that were never possible in their native ranges. Ae. albopictus has largely displaced the longerestablished $A$ e. aegypti in many areas in the southeastern United States, creating complex interactions of competition, cross-mating and evolutionary pressures between these two invasive species (Bargielowski et al. 2013). The ongoing expansion of these two species to new locations in urban and rural communities makes them a central concern for public health. Due to the similarities between these species, control methods are often the same regardless of the presence of one or both species in the area.

\section{Vector control: where, when and who?}

The most familiar stage of the mosquito, the winged adult, is the form that is responsible for disease transmission. However, the mosquito's life cycle involves both aquatic and aerial stages, all of which can be targeted by mosquito control methods for preventing disease. The World Health Organization (WHO) provided the first guidelines for dengue control and prevention, including vector management, in the 1990s, but the importance of Aedes control took a new turn with the strong support given to the Global Vector Control Response by member states during the World Health Assembly in 2017 (WHO 1997, UNICEF/ UNDP/World Bank/WHO 2017). These guidelines include protocols for environmental management of natural and human-made mosquito breeding sites, chemical and biological control agents for treatment of larval and adult stages and best practices for encouraging community engagement (WHO 2012). Mosquito control activities are most successful when multiple approaches are combined and coordinated with other health, environmental and community sectors to produce an integrated approach to mosquito management.

Vector control measures targeting the aquatic larval and pupal stages focus on the removal of human-made containers where Aedes mosquitoes prefer to lay eggs, or these watery habitats can be treated with chemical or biological compounds (i.e. Bacillus thuringiensis) to arrest development or kill immature stages and consequently reduce adult population density (Achee et al. 2015). Preventive measures such as the regular removal of stagnant water (locations A-E, in Figure 14.1) or covering water storage containers are strongly recommended (control measures 1-5 in Figure 14.1). Deployment of larvivorous fish or copepods alone or in combination with other methods has shown low levels of efficacy (Lazaro et al. 2015, Han et al. 2015). 


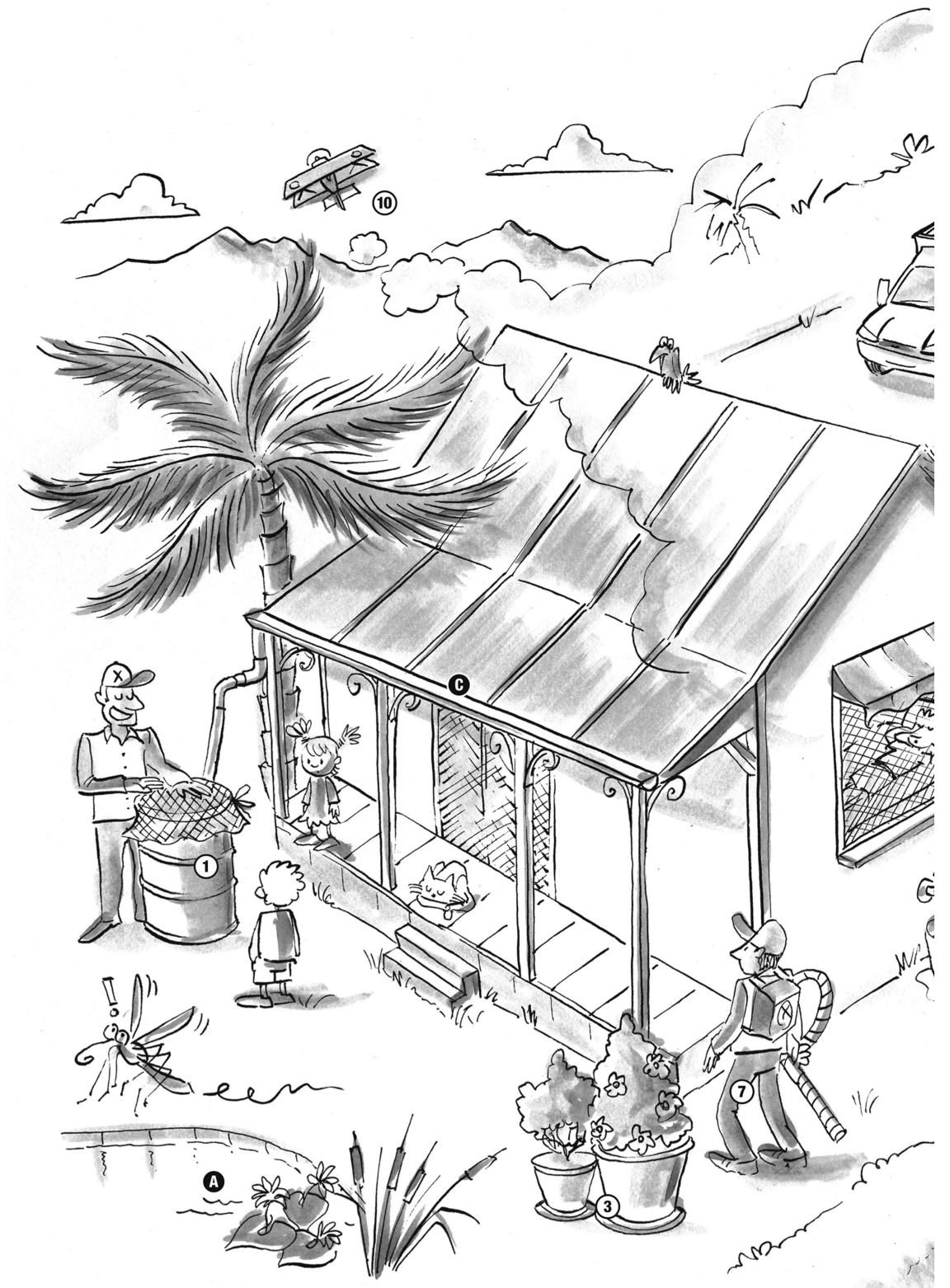

FIGURE 14.1 Suitable habitats for Aedes species and vector control methods. Aedes albopictus and Ae. aegypti breed in human environments. Ae. albopictus also develops in more natural habitats such as ponds, plants that hold water or tree holes (A). Both species are often found breeding in flower pots $(\mathrm{B})$, gutters $(\mathrm{C})$, water containers $(\mathrm{D})$ and water collected in garbage of all sorts such as tyres, fridges and discarded containers (E). To reduce vector-human contact, several measures are used by people and public health authorities. Source reduction eliminates suitable places for females to lay eggs by properly covering 


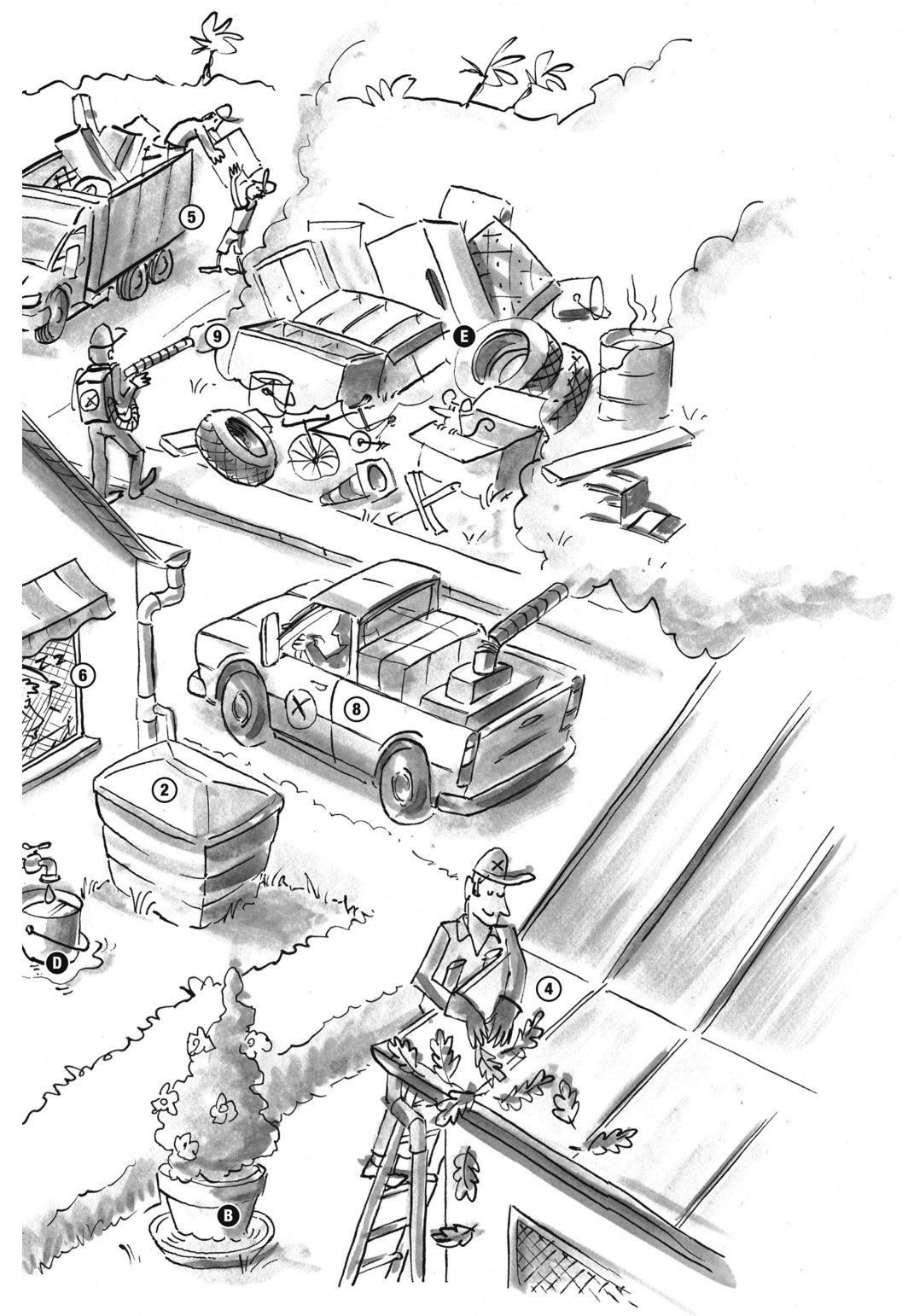

water storage $(1,2)$, removing stagnant water (3), cleaning household premises (4), avoiding garbage accumulation (5) and treating large ponds or reservoirs. To prevent humanvector contact, people at high risk can stay under bed nets even though Aedes mosquitoes are daytime biters, and screening can be installed in windows (6). Additionally, insecticide spatial spraying is performed indoors (7), outdoors (8-9) and occasionally by aircraft (10). The successful control of Aedes mosquitoes is based on the coordinated efforts of communities and public health authorities and by educating the youngest generation to take an active role in prevention (1). Illustration by Vincent Jacquet. 
Interventions against adults aim to kill them in their aerial stage or interrupt female biting to prevent human-vector contact. Measures that target adult fertility and their ability to produce viable eggs aim to reduce future generations that may transmit disease (control measures 6-10 in Figure 14.1). Various adult behaviours can be targeted, including mating, host-seeking, blood-feeding, resting and egg-laying (oviposition). Current Aedes adult control in most countries around the world is based primarily on spraying chemical insecticides formulated for outdoor application via trucks or hand-operated backpacks or in targeted locations where adults can be found resting, such as vegetation for Aedes albopictus or indoor areas for Aedes aegypti (Achee et al. 2015, Faraji and Unlu 2016). Aircraft are also occasionally used as an emergency method (Britch et al. 2018, Likos et al. 2016).

An appropriate combination of vector control measures that target both immature and adult stages of the life cycle is recommended for maximizing density reduction and interrupting eventual virus transmission (Hierlihy et al. 2019). In areas with endemic arbovirus transmission, a low density of mosquitoes is achieved by routine larval control year round, with vector control teams implementing breeding site removal or treatment. During periods of high arbovirus transmission or epidemics, chemical applications, both indoor and outdoor, are used to control adults and reduce mosquito-human contact. Community involvement and participation in reducing breeding sites in urban areas requires sustained education and dedicated social mobilization (location 1, Figure 14.1). A combination of routine surveillance of mosquito breeding activity and disease cases in humans and potential animal hosts is essential for triggering early control strategies for preventing widespread disease transmission.

The decision of when, where and how to control mosquito populations is best made through integrated entomological and epidemiological surveillance as part of a comprehensive management plan (UNICEF/UNDP/World Bank/WHO 2017, Roiz et al. 2018). Without the commitment of political, operational and community stakeholders, such a plan cannot be sustainably developed, validated, funded and implemented (Horstick et al. 2010). Furthermore, local governance and operational policies are structured differently depending on local transmission patterns and the available human capacity and resources. In the end, the successful early interruption of disease transmission depends on interagency preparedness and coordinated actions (Roiz et al. 2018).

\section{Success stories in Aedes aegypti and disease control}

The efficacy of vector control is measured at different steps: lowering density and/or human-vector contact, epidemiological impact and its sustainability over time. There is some evidence that mosquito density can be reduced for a period of time thereby preventing epidemics, but few studies have rigorously demonstrated the long-range efficacy of vector control interventions (Wilson et al. 2015, Bowman et al. 2016). Part of the challenge is demonstrating effectiveness of a 
specific vector control strategy in a treatment area compared to a similar control area with no such intervention. Such experimental protocols are hard to justify during a public health emergency since the control area could be exposed to higher risk of infection and disease. This is part of the reason why clear evidence on the epidemiological impacts of vector control is scarce. However, there are three examples of sustainable success stories in interrupting yellow fever and dengue transmission.

Several reports claim stories of successfully eradicating yellow fever in the early 1900s in the Americas and the Caribbean when the yellow fever virus and its transmission by Aedes aegypti was first described (Soper 1963). Following reports of early successes in reducing urban yellow fever cases and the discovery of DDT (dichlorodiphenyltrichloroethane) as an effective tool in this effort, the Rockefeller Foundation embarked on a worldwide yellow fever and malaria eradication programme in the 1940s (Soper 1963). Combining vaccination with mosquito control, the Rockefeller programme paved the way for modern vector control techniques by relying on large-scale indoor residual spraying of DDT. The effects on Ae. aegypti populations were drastic and the species was believed to be eradicated in the Americas. For a decade no record of either the mosquito or any of the diseases it carried was published.

However, a subsequent progressive recolonization of the mosquito across the continent brought dengue fever and other arboviruses with it (Soper 1963). Pockets of urban yellow fever outbreaks were controlled with vaccination. Malathion and other organophosphorus compounds became the new adulticides of choice, combined with removing larval breeding sites and engaging community through education campaigns. During outbreaks, outdoor spraying of insecticides was favoured over indoor residual spraying.

The return of $A$ e. aegypti was accompanied by an increase in dengue outbreaks, as all four dengue serotypes colonized the Americas. One country was an exception: for 15 years from 1981 to 1997 Cuba managed to remain free of dengue and recorded very low densities of this mosquito. During this period in Cuba an intense programme of surveillance and control was enforced in two phases by combining adult and larval control. The first phase involved massive ultra-low volume (ULV) spraying of malathion by aerial and ground application both indoors and outdoors. Phase two involved nationwide breeding source removal with education programmes for the general population and law enforcement that focused on limiting suitable conditions for larval development in backyards and houses. This combination of vector control methods was accompanied by an emphasis on entomological surveillance, source reduction and larval control (Armada Gessa and Figueredo González 1986). However, in 1997 an increase in vector density, most likely due to weakening in surveillance, along with the introduction of foreign infections, caused the re-emergence of dengue on the island (Kourí et al. 1998).

Another example of success occurred in Singapore during the 1960s, when authorities combined strict larval control with law enforcement after the 
emergence of dengue transmission in the island city-state (Ooi et al. 2006). The programme resulted in low incidence of disease and low entomological indices for 15 years. However, since the 1980s, dengue cases have increased in the city despite low mosquito numbers. Several reasons are hypothesized to explain this disease expansion: the absence of immunity, reduced vector surveillance for case detection, introduction of foreign cases, and possibly a shift in mosquito behaviour.

Aside from these examples, worldwide Aedes vector control efforts have not succeeded in sustainably reducing the arbovirus burden in recent decades.

\section{Reasons for failure: chemical and social}

These experiences present many lessons to inform future surveillance and control strategies. Investigating the reasons for what has failed to sustain long-term effects is crucial to developing strategies for stopping the transmission of Aedesborne diseases. The massive elimination campaigns of the 1940s in the Americas relied heavily on chemical control methods and top-down organization, neither of which are feasible or sustainable today. The authoritarian campaigns were successful for a period of time but led to neglected surveillance and vector control programmes once the vector was thought to be eradicated. Ae. aegypti has now recolonized all of South America, reaching all the way to its southernmost temperature limit in Argentina where it recolonized the capital, Buenos Aires, in 1991 (Zanotti et al. 2015).

With our current understanding of the long-term environmental consequences of chemical insecticides, the mass spraying of DDT and similar materials is no longer an acceptable strategy, and not only because of environmental concerns. Indeed, chemical control methods for both larvae and adults are reaching the end of their effective use for sustainable control because Aedes populations worldwide are becoming resistant to a wide range of compounds. Early evidence of resistance was found with DDT, followed by resistances to a range of organophosphates including the larvicide temephos (Moyes et al. 2017). Malathion remains a useful compound for mosquito control, but its toxicity to mammals restricts its use. The biological insecticide Bacillus thuringiensis var. israelensis, for which no resistance has yet been observed, has largely replaced temephos.

Pyrethroids, then, have become the insecticide of choice: inexpensive, harmless to wildlife, and applicable indoors as spatial and residual spray, outdoors as ultra-low volume (UVL) aerosol, and even impregnated in cloth material. These advantages are leading to a monotherapy conducive to the widespread development of resistance (Moyes et al. 2017). In addition, since 2010, pyrethroids are the only approved compounds for adult insect control in the European Union. In the absence of any alternative, some areas such as French overseas territories have arrived at a chemical control dead-end.

Aside from the widespread mosquito resistance, the method of spraying large quantities of insecticide in the environment is itself controversial because of the 
lack of evidence for efficacy, high cost, slow operational response, low community acceptance, and the potential impacts on non-target organisms in the environment (Esu et al. 2010, Knauer et al. 2017). Selective pressures exerted on Aedes populations from vector control are compounded when the same pyrethroid insecticides are used against pest mosquitoes, found in household insecticides and impregnated gardening and personal protection materials, driving pyrethroid resistance even further and possibly preventing its reversal (Macoris et al. 2018, Gray et al. 2018).

We have arrived at the point where both chemicals and the methods for applying them may be ineffective for controlling Aedes, with few acceptable alternatives existing. At the very least, chemical applications for emergency tools could be regained by mandating non-chemical alternatives for non-emergency situations, or by developing novel compounds that target the vectors with other modes of action and that are more selective for mosquitoes (Dusfour et al. 2019). In the absence of novel tools that are validated, recommended or available, one possibility is to increase the use of pyrethroid-impregnated materials (in window curtains, for example) and the reinstatement of indoor residual spraying with pyrethroids (Samuel et al. 2017, Banks et al. 2015). These options are less harmful for the environment as they are localized to the indoors and effective against non-resistant strains of Ae. aegypti which prefer to rest indoors. For Ae. albopictus mosquitoes that tend to rest outside households, these alternatives would not be effective. In the absence of efficient compounds against adults, the only other effective options are the application of larvicides or the alteration of larval breeding sites for large-scale mosquito population control. Typically, water collection areas or containers are drained to eliminate putative breeding sites, covered to prevent egg-laying, filled in with sand to keep moisture for gardens without stagnant water or manipulated in such a way that mosquito larvae cannot develop or grow.

Because Aedes females prefer to lay eggs in small human-made containers of water, households (especially those without reliable piped water) are an important source of breeding grounds for the mosquito. Community engagement, therefore, is essential for comprehensive larval control. However, such engagement has not shown long-lasting success outside of the Cuban and Singaporean examples, both of which involved strong, authoritarian enforcement. Even though education and promotion plans were integrated into strategies, topdown approaches push the population to rely on authorities and to ignore their own personal role and responsibility in source reduction (Perez-Guerra et al. 2009, Mieulet and Claeys 2014). This behaviour is exacerbated by the belief that neighbours are not doing enough and individual efforts are made in vain (Ibarra et al. 2014). The mosquito is generally and universally hated mainly because of its bite: people are often more motivated by the nuisance they cause than by diseases they transmit (Dickinson and Paskewitz 2012). Reducing Aedes breeding sites, however, does not always have a direct and noticeable effect on the perception of overall nuisance, since bites may continue even from a diminished population or from other mosquito species. In contexts where other, more critical health 
and safety concerns dominate, mosquito-borne diseases may not take priority in the lives of the community so that communication tools developed by vector control authorities may be ignored or misunderstood (Mieulet and Claeys 2014, Anderson et al. 2020). Being sensitive to local beliefs and instilling a basic understanding of disease transmission and the mosquito life cycle are also critical for mobilizing the population to act as participants in mosquito control and participate in bottom-up interventions (Ibarra et al. 2014, Paz-Soldán et al. 2011, Frank et al. 2017). Developing effective social strategies to support vector control strategies has therefore become a key recommendation and the WHO has published recommendations for guiding and supporting socially sensitive vector control teams (Bartumeus et al. 2019, Parks and Lloyd 2005).

A team of vector control specialists cannot possibly monitor all potential breeding sites when larvae can develop into adults in only two weeks under optimal temperature conditions; therefore, involving communities in control and surveillance is essential for efficiently covering or disrupting all possible breeding sites (Gubler and Clark 1996). Successful examples of community engagement also highlight the importance of regular surveillance protocols to monitor mosquito activity, disease incidence and mosquito resistance and then sustain these efforts over time (Bardach et al. 2019, Sulistyawati et al. 2019). This integrated approach has been advocated for decades but requires intensive and constant efforts from all stakeholders, even during periods when there is no disease transmission. The best results require political commitment, sustainable allocation of resources for planning and surveillance, as well as the training of public health authorities as part of an integrated and holistic approach for mosquito control. Despite decades of Aedes vector control experiences, widespread comprehensive and sustained strategies of mosquito control are not currently the norm in most endemic areas (Roiz et al. 2018). As a consequence, sound strategies have by and large failed to be implemented or sustained (Gubler 2005, Roiz et al. 2018, WHOPES 2010).

In the face of dramatic recent increases in dengue and other arboviruses, few success stories and little solid evidence for effective mosquito control, many questions are left open about the future prevention of vector-borne diseases (Bowman et al. 2016). For decades, the challenges and the calls for action have been stated in publications and reports-yet dengue remains a neglected disease. With a 30 -fold increase in the past 50 years, dengue is finally being taken seriously (UNICEF/UNDP/World Bank/WHO 2017, WHO 2012). The re-emergence of yellow fever, the Zika outbreak and the emergence of other novel sylvatic arboviruses carry a warning to authorities and the public about an imminent threat. Aedes-borne arbovirus transmission is finally drawing the attention of researchers to develop new tools for surveillance and control.

\section{The future of control against Aedes and disease}

The global community is facing new challenges in controlling arboviruses. At the same time, modern vector control is still placed firmly in the dream 
of past successes, expecting old tools and old methods to be efficient against new diseases, impacting only the targeted vector, while being environmentally friendly, sustainable and acceptable by communities all over the globe. Clearly, new tools and strategies are urgently needed.

As the number of available and effective compounds continues to decrease, it is of primary importance to regain the efficacy of pyrethroids and avoid further development of resistance. Integrated vector control must include monitoring insecticide resistance and measuring current insecticide efficacy in a comprehensive plan (Dusfour et al. 2019). Available control compounds are scarce but remain crucial for emergency control during outbreaks. As mentioned earlier, the efficacy of spatial sprays is debatable but pyrethroids could be used for indoor residual spraying or impregnation of materials. To reduce the selection pressure for resistance, alternative tools must be developed, validated and deployed.

To ensure the quality and effectiveness of proposed products and tools, the WHO has established the Prequalification Team (PQT), which replaces the WHO Pesticide Evaluation Scheme (WHOPES). The PQT supports the development, evaluation and adoption of novel control methods. An independent Vector Control Advisory Group provides additional guidance to product developers, innovators and researchers, including guidelines on the acquisition of epidemiological data, study design and new vector interventions. This group also provides advice to the WHO Strategic and Technical Advisory Group for neglected tropical diseases.

Based on the failure to maintain long-term successes in past control efforts and the expanded knowledge that the research and vector control communities have accumulated over the years, a more integrated view of vector strategies and technological advances has led to a suite of novel tools and new methods to implement them. While professional teams are still mainly responsible for implementation, the general public is now involved at an early stage, becoming an obvious and necessary component to establish and sustain vector control knowledge and practices in the affected communities (Kolopack et al. 2015, Ernst et al. 2015). Research in citizen and social sciences such as anthropology are also accompanying the expanded use of social networks and integrating mobile and geospatial technologies for providing new potential for vector control (Sousa et al. 2017, Hamer et al. 2018). Better understanding of the community's perception, knowledge, practices, beliefs and reluctance/acceptance of mosquito control is crucial for developing appropriate local communication and media messages (McNaughton 2012). Education must be sustainably implemented beginning at a young age, and adapted to local beliefs, habits and infrastructure. People must mobilize not only for vector control but also for surveillance in cooperation with a coordinated public health framework.

The research and development of new tools for targeting vector species is revealing more efficient and environmentally friendly techniques. One category is based on the knowledge of the vector's biology, behaviour and ecology to trap 
or otherwise prevent it from coming into contact with people. For example, there are autodissemination traps, which exploit the cryptic behaviour of oviposition, when adult mosquitoes pick up a residue of larvicide that they then spread to other breeding sites (Maoz et al. 2017). Toxic baits that contain a sugarinsecticide compound target behaviours of both adult male and female sugarfeeding (Revay et al. 2014). Those tools are currently under evaluation, and even though some have proven their efficacy in reducing mosquito density, none have yet demonstrated epidemiological successes. These tools are relatively easy to implement and can be widely distributed, although their chemical composition may require authorization in areas where they are not commercially available. The fact that such tools rely on community involvement may factor into their chances of success (Faraji and Unlu 2016). Other tools such as the trapping of large numbers of adults through attractant compounds and behaviour-modifying compounds have shown promise in lab tests. However, better attractant compounds are still needed to demonstrate significant reductions in mosquito density (Degener et al. 2015, Obermayr et al. 2015).

The second category of novel control technologies relies on genetic modification (GM) of the mosquito (Qsim et al. 2017). The objective here is to produce non-fertilized eggs or non-viable offspring, thereby reducing the density of future generations. The sterile insect technique can be achieved through several methods. One method focuses on producing sterile males by irradiation (SIT); another relies on genetically modified mosquitoes to carry a lethal gene (RIDL); a third method utilizes insect incompatibilities with Wolbachia-modified mosquitoes (IIT) (Crawford et al. 2020, Kittayapong et al. 2019, Thomas et al. 2000). The last has the advantage of inhibiting arbovirus multiplication and interrupting its transmission (Ryan et al. 2019). In addition, the RNA interference technique is being tested for mosquito control as both SIT and insecticidal tools (Giesbrecht et al. 2020). Whether alone or in combination, SIT, RIDL and IIT mosquitoes have proven to be of some efficacy in controlling insects in field trials, but so far such techniques have shown only preliminary evidence for controlling disease (Crawford et al. 2020, Bellini et al. 2013, Carvalho et al. 2015, Kittayapong et al. 2019).

The general public and government agencies are often sceptical about genetically modified or altered mosquito technologies and more evidence for their efficacy is needed before they can become part of a public campaign to improve their acceptance (Ernst et al. 2015, Kolopack et al. 2015). Such concerns were heightened with the discovery in Brazil that genetically modified Ae. aegypti transferred some of their GM genes into the wild population (Evans et al. 2019). In Europe, GM mosquitoes are highly regulated and access to such technology is controlled and limited. Because mosquitoes irradiated to produce sterile offspring (SIT) are not considered genetically modified, some have been used and tested in Italy and in Reunion Island, France. Guidelines and principles for evaluating fertility-altered mosquitoes are different from one country to another (Panjwani and Wilson 2016). In any case, all methods targeting mosquito fertility entail 
significant costs to maintain dedicated infrastructure and personnel for longterm mosquito mass-rearing and release (Meghani and Boëte 2018). At present, none of the new tools are fully validated or widely available, leaving traditional insecticides and source reduction methods as the sole pillars for controlling mosquitoes in the context of disease epidemics.

\section{Zoonotic and epizootic mosquito control}

While urban vectors and their associated arboviruses are at the centre of control efforts in both temperate and tropical areas, a growing concern is arising around zoonotic diseases. Viruses (and other pathogens) cycle between forestdwelling mosquitoes and wild animals. Viruses such as Zika can be transmitted to humans when the virus enters an urban cycle with Aedes mosquitoes or it can be aided by bridge mosquitoes like Ae. albopictus that move between rural and urban habitats (Pereira et al. 2020). Many of these viruses infect humans as accidental and dead-end hosts, meaning that the virus may infect a human but is insufficiently amplified in the human body to be transmitted to another mosquito (Weaver and Reisen 2010, Wilder-Smith et al. 2017). Such is the case for West Nile virus, Usutu, Eastern equine encephalitis and Serogroup California viruses, which have all attracted attention in Europe and North America in the last few years (Gill et al. 2019, Lindsey et al. 2020, Vilibic-Cavlek et al. 2019, Calzolari et al. 2020). West Nile virus is of particular concern, and has received more research since its arrival and expansion in North America in the late 1990 s.

With the emergence of more viruses using humans as dead-end hosts, controlling transmission has become an important challenge. The culprit mosquito species do not all belong to the genus Aedes, with Coquillettidia, Culex and Culiseta also implicated, mosquitoes with vastly different ecologies and some already recognized as nuisance pests (Sherwood et al. 2020, Hesson et al. 2019, Martinet et al. 2019). Several of these latter mosquito species transmit viruses to humans with varying abilities. Their physiological and ecological requirements are as different as are their life cycles over the seasons. Unlike Aedes, few are container-breeders, for example, creating complications for integrated vector control strategies in areas they coinhabit with other vectors. Such mosquitoes do not transmit disease as readily in their urban cycles, with the effect that there is not as much research about ways to include their habits in integrated vector control plans.

Controlling these other vector mosquitoes therefore presents difficult challenges. Personal protection and prevention measures such as topical repellents are recommended along with larval control; controlling adults or releasing sterile males are not recommended against West Nile virus (Hongoh et al. 2016, Campagna, Trudel and INSPQ 2018, CDC 2019b). Reduction of West Nile vectors by larvicides or adulticides often depends on mass spraying, a decidedly old-fashioned technique. Strategies tend to be implemented 
in emergency mode relying on tools already in place for controlling nuisance or other vector mosquitoes (CDC 2019a, Werth 2019). With such outbreaks occurring with increasing frequency and greater severity, the means of controlling target mosquitoes or otherwise reducing disease transmission should be carried out with consideration for environmental impacts. To complicate matters, some of these species are already resistant to insecticides, leaving their efficacy unproven in both reducing mosquito numbers and transmitting diseases (Scott et al. 2015, Dunbar et al. 2018). Novel control methods need to be developed but the knowledge of these species is scarce, leaving the first-line strategy one of heightening people's awareness of using personal protections and developing efficient surveillance tools (Kading et al. 2020, Hongoh et al. 2016, Lindsey et al. 2020).

\section{Conclusion}

Since the discovery of the mosquito's capacity to transmit pathogens that cause diseases, humanity has tried to control these vectors to reduce the disease burden. The vectorial systems involve hosts, vectors and pathogens in the natural environment in a complex interplay that is constantly evolving under selective pressures. To reach a point where people and mosquitoes can achieve a sustainable and acceptable equilibrium that simultaneously preserves human health and protects the environment, one must aim to integrate all aspects of the ecology of mosquito-borne disease with the habits of the few mosquitoes that transmit those diseases. Just as the Aedes aegypti mosquito has fully adapted to living with humans, people must learn to adapt their own habits and urban environments to minimize their exposure to this and other dangerous mosquitoes. Targeted and integrated approaches for reducing the transmission of urban mosquito diseases have been advocated for decades but are unevenly applied due to their costs, limited human capacity, community apathy and weakness of the political will that is required to sustain these efforts during interepidemic periods. With the rising threat of mosquito-borne diseases, these approaches must be strengthened and adapted if we want to reduce pathogen transmission.

\section{Bibliography}

Achee, Nicole L., Fred Gould, T. Alex Perkins, Robert C. Reiner Jr, Amy C. Morrison, Scott A. Ritchie, Duane J. Gubler, Remy Teyssou, and Thomas W. Scott. 2015. A Critical Assessment of Vector Control for Dengue Prevention. PLOS Neglected Tropical Diseases 9 (5): e0003655. https://doi.org/10.1371/journal.pntd.0003655.

Anderson, Elizabeth J., Kacey C. Ernst, Francisco Fernando Martins, Cicera da Silva Martins, and Mary P. Koss. 2020. Women's Health Perceptions and Beliefs Related to Zika Virus Exposure during the 2016 Outbreak in Northern Brazil. The American Journal of Tropical Medicine and Hygiene 102 (3): 629-633. https://doi.org/10.4269/ ajtmh.19-0311. 
Armada Gessa, J.A., and R. Figueredo González. 1986. Application of Environmental Management Principles in the Program for Eradication of Aedes (Stegomyia) aegypti (Linneus, 1762) in the Republic of Cuba, 1984. Bulletin of the Pan American Health Organization 20 (2): 186-193.

Banks, Sarah DeRaedt, James Orsborne, Salvador A. Gezan, Harparkash Kaur, Annelies Wilder-Smith, Steve W. Lindsey, and James G. Logan. 2015. Permethrin-Treated Clothing as Protection against the Dengue Vector, Aedes aegypti: Extent and Duration of Protection. PLOS Neglected Tropical Diseases 9 (10): e0004109. https://doi.org/10.1 371/journal.pntd.0004109.

Bardach, Ariel Esteban, Herney Andrés García-Perdomo, Andrea Alcaraz, Elena Tapia López, Ruth Amanda Ruano Gándara, Silvina Ruvinsky, and Agustín Ciapponi. 2019. Interventions for the Control of Aedes aegypti in Latin America and the Caribbean: Systematic Review and Meta-Analysis. Tropical Medicine E International Health: 24 (5): 530-552. https://doi.org/10.1111/tmi.13217.

Bargielowski, Irka E., L. Philip Lounibos, and María Cristina Carrasquilla. 2013. Evolution of Resistance to Satyrization through Reproductive Character Displacement in Populations of Invasive Dengue Vectors. Proceedings of the National Academy of Sciences of the United States of America 110 (8): 2888-2892.

Bartumeus, Frederic, Guilherme B. Costa, Roger Eritja, Ann H Kelly, Marceline Finda, Javier Lezaun, Fredros Okumu, et al. 2019. Sustainable Innovation in Vector Control Requires Strong Partnerships with Communities. PLoS Neglected Tropical Diseases 13 (4). https://journals.plos.org/plosntds/article?id=10.1371/journal.pntd.0007204.

Bellini, R., A. Medici, A. Puggioli, F. Balestrino, and M. Carrieri. 2013. Pilot Field Trials with Aedes albopictus Irradiated Sterile Males in Italian Urban Areas. Journal of Medical Entomology 50 (2): 317-325. https://doi.org/10.1603/me12048.

Bowman, Leigh R., Sarah Donegan, and Philip J. McCall. 2016. Is Dengue Vector Control Deficient in Effectiveness or Evidence?: Systematic Review and MetaAnalysis. PLoS Neglected Tropical Diseases 10 (3): e0004551. https://doi.org/10.1371/j ournal.pntd.0004551.

Bradshaw, Corey J.A., Boris Leroy, Céline Bellard, David Roiz, Céline Albert, Alice Fournier, Morgane Barbet-Massin, Jean-Michel Salles, Frédéric Simard, and Franck Courchamp. 2016. Massive yet Grossly Underestimated Global Costs of Invasive Insects. Nature Communications 7 (1): 12986. https://doi.org/10.1038/ncomms12986.

Britch, S.C., K.J. Linthicum, R.L. Aldridge, M.S. Breidenbaugh, M.D. Latham, P.H. Connelly, and M.J.E. Rush, J.L. Remmers, J.D. Kerce, C.A. Silcox. 2018. US Navy Entomology Center of Excellence Team. Aerial ULV control of Aedes aegypti with naled (Dibrom) inside simulated rural village and urban cryptic habitats. PLoS One 13 (1): e0191555. doi: 10.1371/journal.pone.0191555. PMID: 29352307; PMCID: PMC5774805.

Calzolari, Mattia, Paola Angelini, Luca Bolzoni, Paolo Bonilauri, Roberto Cagarelli, Sabrina Canziani, Danilo Cereda, et al. 2020. Enhanced West Nile Virus Circulation in the Emilia-Romagna and Lombardy Regions (Northern Italy) in 2018 Detected by Entomological Surveillance. Frontiers in Veterinary Science 7: 243. https://doi.org/10 $.3389 /$ fvets.2020.00243.

Campagna, Céline, Richard Trudel, and INSPQ. 2018. Évaluation de l'efficacité des larvicides contre les espèces vectrices du virus du Nil occidental: Rapport d'évaluation. http://collections .banq.qc.ca/ark:/52327/3579857.

Carvalho, Danilo O., Andrew R. McKemey, Luiza Garziera, Renaud Lacroix, Christl A. Donnelly, Luke Alphey, Aldo Malavasi, and Margareth L. Capurro. 2015. Suppression of a Field Population of Aedes aegypti in Brazil by Sustained Release of Transgenic 
Male Mosquitoes. PLoS Neglected Tropical Diseases 9 (7): e0003864. https://doi.org/10 .1371/journal.pntd.0003864.

CDC. 2019a. Information on Aerial Spraying. Centers for Disease Control. October 2. https ://www.cdc.gov/easternequineencephalitis/mosquitocontrol/aerial-spraying.html.

CDC. 2019b. Prevention. October 7. https://www.cdc.gov/lac/gen/pre.html.

Crawford, Jacob E., David W. Clarke, Victor Criswell, Mark Desnoyer, Devon Cornel, Brittany Deegan, Kyle Gong, et al. 2020. Efficient Production of Male Wolbachia -Infected Aedes aegypti Mosquitoes Enables Large-Scale Suppression of Wild Populations. Nature Biotechnology 38 (4): 482-492. https://doi.org/10.1038/s41587 -020-0471-x.

Degener, Carolin Marlen, Tatiana Mingote Ferreira de Ázara, Rosemary Aparecida Roque, Susanne Rösner, Eliseu Soares Oliveira Rocha, Erna Geessien Kroon, Cláudia Torres Codeço, et al. 2015. Mass Trapping with MosquiTRAPs Does Not Reduce Aedes aegypti Abundance. Memórias Do Instituto Oswaldo Cruz 110 (4): 517527. https://doi.org/10.1590/0074-02760140374.

Dickinson, Katherine, and Susan Paskewitz. 2012. Willingness to Pay for Mosquito Control: How Important Is West Nile Virus Risk Compared to the Nuisance of Mosquitoes? Vector Borne and Zoonotic Diseases 12 (10): 886-892.

Dunbar, Mike W., Amanda Bachmann, and Adam J. Varenhorst. 2018. Reduced Insecticide Susceptibility in Aedes vexans (Diptera: Culicidae) Where Agricultural Pest Management Overlaps With Mosquito Abatement. Journal of Medical Entomology 55 (3): 747-751. https://doi.org/10.1093/jme/tjx245.

Dusfour, Isabelle, John Vontas, Jean-Philippe David, David Weetman, Dina M. Fonseca, Vincent Corbel, Kamaraju Raghavendra, et al. 2019. Management of Insecticide Resistance in the Major Aedes Vectors of Arboviruses: Advances and Challenges. PLoS Neglected Tropical Diseases 13 (10): e0007615. https://doi.org/10.1371/journal .pntd.0007615.

Ernst, Kacey C., Steven Haenchen, Katherine Dickinson, Michael S. Doyle, Kathleen Walker, Andrew J. Monaghan, and Mary H. Hayden. 2015. Awareness and Support of Release of Genetically Modified 'Sterile' Mosquitoes, Key West, Florida, USA. Emerging Infectious Diseases 21 (2): 320-324. https://doi.org/10.3201/eid2102.141035.

Esu, E., A. Lenhart, L. Smith, and O. Horstick. 2010. Effectiveness of Peridomestic Space Spraying with Insecticide on Dengue Transmission; Systematic Review. Trop Med Int Health 15 (May): 619-631. https://doi.org/10.1111/j.1365-3156.2010.02489.x.

Evans, Benjamin R., Panayiota Kotsakiozi, Andre Luis Costa-da-Silva, Rafaella Sayuri Ioshino, Luiza Garziera, Michele C. Pedrosa, Aldo Malavasi, Jair F. Virginio, Margareth L. Capurro, and Jeffrey R. Powell. 2019. Transgenic Aedes aegypti Mosquitoes Transfer Genes into a Natural Population. Scientific Reports 9 (1): 13047. https://doi.org/10.1038/s41598-019-49660-6.

Faraji, Ary, and Isik Unlu. 2016. The Eye of the Tiger, the Thrill of the Fight: Effective Larval and Adult Control Measures Against the Asian Tiger Mosquito, Aedes Albopictus (Diptera: Culicidae), in North America. Journal of Medical Entomology 53 (5): 1029-1047. https://doi.org/10.1093/jme/tjw096.

Frank, Amy L, Emily R Beales, Gilles de Wildt, Graciela Meza Sanchez, and Laura L Jones. 2017. "We Need People to Collaborate Together against This Disease": A Qualitative Exploration of Perceptions of Dengue Fever Control in Caregivers' of Children under 5 Years, in the Peruvian Amazon. PLoS Neglected Tropical Diseases 11 (9): e0005755.

Giesbrecht, David, Daniel Heschuk, Ian Wiens, David Boguski, Parker LaChance, and Steve Whyard. 2020. RNA Interference Is Enhanced by Knockdown of 
Double-Stranded RNases in the Yellow Fever Mosquito Aedes aegypti. Insects 11 (6). https://doi.org/10.3390/insects11060327.

Gill, Christine M., J. David Beckham, Amanda L. Piquet, Kenneth L. Tyler, and Daniel M. Pastula. 2019. Five Emerging Neuroinvasive Arboviral Diseases: Cache Valley, Eastern Equine Encephalitis, Jamestown Canyon, Powassan, and Usutu. Seminars in Neurology 39 (4): 419-427. https://doi.org/10.1055/s-0039-1687839.

Gray, Lyndsey, Sergio Dzib Florez, Anuar Medina Barreiro, José Vadillo-Sánchez, Gabriela González-Olvera, Audrey Lenhart, Pablo Manrique-Saide, and Gonzalo M. Vazquez-Prokopec. 2018. Experimental Evaluation of the Impact of Household Aerosolized Insecticides on Pyrethroid Resistant Aedes aegypti. Scientific Reports 8 (August). https://doi.org/10.1038/s41598-018-30968-8.

Gubler, D.J.. 2005. The Emergence of Epidemic Dengue Fever and Dengue Hemorrhagic Fever in the Americas: A Case of Failed Public Health Policy. Revista Panamericana de Salud Pública 17 (4). https://doi.org/10.1590/S1020-49892005000400001.

Gubler, D.J., and G.G. Clark. 1996. Community Involvement in the Control of Aedes aegypti. Acta Tropica 61 (2): 169-179. https://doi.org/10.1016/0001-706x(95)00103-1.

Hamer, Sarah A., Rachel Curtis-Robles, and Gabriel L. Hamer. 2018. Contributions of Citizen Scientists to Arthropod Vector Data in the Age of Digital Epidemiology. Current Opinion in Insect Science 28: 98-104. https://doi.org/10.1016/j.cois.2018.05.005.

Han, W.W., A. Lazaro, P.J. McCall, L. George, S. Runge-Ranzinger, J. Toledo, R. Velayudhan, and O. Horstick. 2015. Efficacy and Community Effectiveness of Larvivorous Fish for Dengue Vector Control. Tropical Medicine \& International Health 20 (9): 1239-1256. https://doi.org/10.1111/tmi.12538.

Hesson, Jenny C., Emma Lundin, Åke Lundkvist, and Jan O. Lundström. 2019. Surveillance of Mosquito Vectors in Southern Sweden for Flaviviruses and Sindbis Virus. Infection Ecology \& Epidemiology 9 (1): 1698903. https://doi.org/10.1080/2 0008686.2019.1698903.

Hierlihy, Catherine, Lisa Waddell, Ian Young, Judy Greig, Tricia Corrin, and Mariola Mascarenhas. 2019. A Systematic Review of Individual and Community Mitigation Measures for Prevention and Control of Chikungunya Virus. PloS One 14 (2): e0212054. https://doi.org/10.1371/journal.pone.0212054.

Hongoh, Valerie, Céline Campagna, Mirna Panic, Onil Samuel, Pierre Gosselin, JeanPhilippe Waaub, André Ravel, Karim Samoura, and Pascal Michel. 2016. Assessing Interventions to Manage West Nile Virus Using Multi-Criteria Decision Analysis with Risk Scenarios. PLOS ONE 11 (8): e0160651. https://doi.org/10.1371/journal .pone.0160651.

Horstick, Olaf, Silvia Runge-Ranzinger, Michael B. Nathan, and Axel Kroeger. 2010. Dengue Vector-Control Services: How Do They Work? A Systematic Literature Review and Country Case Studies. Transactions of the Royal Society of Tropical Medicine and Hygiene 104 (6): 379-386. https://doi.org/10.1016/j.trstmh.2009.07.027.

Ibarra, Anna M Stewart, Valerie A Luzadis, Mercy J Borbor Cordova, Mercy Silva, Tania Ordoñez, Efraín Beltrán Ayala, and Sadie J Ryan. 2014. A Social-Ecological Analysis of Community Perceptions of Dengue Fever and Aedes aegypti in Machala, Ecuador. BMC Public Health 14 (1): 1135.

Kading, Rebekah C., Lee W. Cohnstaedt, Ken Fall, and Gabriel L. Hamer. 2020. Emergence of Arboviruses in the United States: The Boom and Bust of Funding, Innovation, and Capacity. Tropical Medicine and Infectious Disease 5 (2). https://doi.org /10.3390/tropicalmed5020096.

Kittayapong, Pattamaporn, Suwannapa Ninphanomchai, Wanitch Limohpasmanee, Chitti Chansang, Uruyakorn Chansang, and Piti Mongkalangoon. 2019. Combined 
Sterile Insect Technique and Incompatible Insect Technique: The First Proof-ofConcept to Suppress Aedes aegypti Vector Populations in Semi-Rural Settings in Thailand. PLOS Neglected Tropical Diseases 13 (10): e0007771. https://doi.org/10.1371 /journal.pntd.0007771.

Knauer, Katja, Nadzeya Homazava, Marion Junghans, and Inge Werner. 2017. The Influence of Particles on Bioavailability and Toxicity of Pesticides in Surface Water. Integrated Environmental Assessment and Management 13 (4): 585-600. https://doi.org /10.1002/ieam.1867.

Kolopack, Pamela A, Janet A Parsons, and James V Lavery. 2015. What Makes Community Engagement Effective?: Lessons from the Eliminate Dengue Program in Queensland Australia. PLoS Neglected Tropical Diseases 9 (4). https://www.ncbi.nlm.nih.gov/pmc /articles/PMC4395388/.

Kourí, Gustavo, María Guadalupe Guzmán, Luis Valdés, Isabel Carbonel, Delfina del Rosario, Susana Vazquez, José Laferté, Jorge Delgado, and María V. Cabrera. 1998. Reemergence of Dengue in Cuba: A 1997 Epidemic in Santiago de Cuba. Emerging Infectious Diseases 4 (1). https://doi.org/10.3201/eid0401.980111.

Kraemer, Moritz U.G., Robert C. Reiner, Oliver J. Brady, Jane P. Messina, Marius Gilbert, David M. Pigott, Dingdong Yi, et al. 2019. Past and Future Spread of the Arbovirus Vectors Aedes aegypti and Aedes albopictus. Nature Microbiology 4 (5): 854863. https://doi.org/10.1038/s41564-019-0376-y.

Lazaro, A., W.W. Han, P. Manrique-Saide, L. George, R. Velayudhan, J. Toledo, S. Runge Ranzinger, and O. Horstick. 2015. Community Effectiveness of Copepods for Dengue Vector Control: Systematic Review. Tropical Medicine \& International Health 20 (6): 685-706. https://doi.org/10.1111/tmi.12485.

Likos, Anna, Isabel Griffin, Andrea M. Bingham, Danielle Stanek, Marc Fischer, Stephen White, Janet Hamilton, et al. 2016. Local Mosquito-Borne Transmission of Zika Virus - Miami-Dade and Broward Counties, Florida, June-August 2016. MMWR. Morbidity and Mortality Weekly Report 65 (38): 1032-1038. https://doi.org/10.2307/ 24858999.

Lindsey, Nicole P., Stacey W. Martin, J. Erin Staples, and Marc Fischer. 2020. Notes from the Field: Multistate Outbreak of Eastern Equine Encephalitis Virus — United States, 2019. MMWR. Morbidity and Mortality Weekly Report 69 (2): 50-51. https://doi.org/10 .15585/mmwr.mm6902a4.

Macoris, Maria de Lourdes, Ademir Jesus Martins, Maria Teresa Macoris Andrighetti, José Bento Pereira Lima, and Denise Valle. 2018. Pyrethroid Resistance Persists after Ten Years Without Usage against Aedes aegypti in Governmental Campaigns: Lessons from São Paulo State, Brazil. PLoS Neglected Tropical Diseases 12 (3): e0006390. https ://doi.org/10.1371/journal.pntd.0006390.

Maoz, Dorit, Tara Ward, Moody Samuel, Pie Müller, Silvia Runge-Ranzinger, Joao Toledo, Ross Boyce, Raman Velayudhan, and Olaf Horstick. 2017. Community Effectiveness of Pyriproxyfen as a Dengue Vector Control Method: A Systematic Review. PLoS Neglected Tropical Diseases 11 (7): e0005651. https://doi.org/10.1371/j ournal.pntd.0005651.

Marcondes, Carlos Brisola. 2019. Medical and Veterinary Entomology. Atheneu. Rio de Janairo: Elsevier. https://doi.org/10.1016/C2017-0-00210-0.

Martinet, Jean-Philippe, Hubert Ferté, Anna-Bella Failloux, Francis Schaffner, and Jérôme Depaquit. 2019. Mosquitoes of North-Western Europe as Potential Vectors of Arboviruses: A Review. Viruses 11 (11). https://doi.org/10.3390/v11111059.

McNaughton, Darlene. 2012. The Importance of Long-Term Social Research in Enabling Participation and Developing Engagement Strategies for New Dengue 
Control Technologies. PLOS Neglected Tropical Diseases 6 (8): e1785. https://doi.org /10.1371/journal.pntd.0001785.

Meghani, Zahra, and Christophe Boëte. 2018. Genetically Engineered Mosquitoes, Zika and Other Arboviruses, Community Engagement, Costs, and Patents: Ethical Issues. PLoS Neglected Tropical Diseases 12 (7): e0006501. https://doi.org/10.1371/journal.pnt d.0006501.

Mieulet, Elise, and Cécilia Claeys. 2014. The Implementation and Reception of Policies for Preventing Dengue Fever Epidemics: A Comparative Study of Martinique and French Guyana. Health, Risk \& Society 16 (7-8): 581-599. https://doi.org/10.1080/1 3698575.2014 .949224$.

Moyes, Catherine L., John Vontas, Ademir J. Martins, Lee Ching Ng, Sin Ying Koou, Isabelle Dusfour, Kamaraju Raghavendra, et al. 2017. Contemporary Status of Insecticide Resistance in the Major Aedes Vectors of Arboviruses Infecting Humans. PLoS Neglected Tropical Diseases 11 (7): e0005625. https://doi.org/10.1371/journal.pnt d.0005625.

Obermayr, Ulla, Joachim Ruther, Ulrich R. Bernier, Andreas Rose, and Martin Geier. 2015. Evaluation of a Push-Pull Approach for Aedes aegypti (L.) Using a Novel Dispensing System for Spatial Repellents in the Laboratory and in a Semi-Field Environment. PLoS ONE 10 (6). https://doi.org/10.1371/journal.pone.0129878.

Ooi, Eng-Eong, Kee-Tai Goh, and Duane J. Gubler. 2006. Dengue Prevention and 35 Years of Vector Control in Singapore. Emerging Infectious Diseases 12 (6). https://doi .org/10.3201/eid1206.051210.

Panjwani, Anusha, and Anthony Wilson. 2016. What Is Stopping the Use of Genetically Modified Insects for Disease Control? PLOS Pathogens 12 (10): e1005830. https://doi .org/10.1371/journal.ppat.1005830.

Parks, Will, and Linda Lloyd. 2005. Planning Social Mobilization and Communication for Dengue Fever Prevention and Control: A Step-by-Step Guide. Geneva: World Health Organization.

Paupy, C., H. Delatte, L. Bagny, V. Corbel, and D. Fontenille. 2009. Aedes Albopictus, an Arbovirus Vector: From the Darkness to the Light. Microbes Infect 11 (December): 1177-1185. https://doi.org/10.1016/j.micinf.2009.05.005.

Paz-Soldan, Valerie A., Valaikanya Plasai, Amy C. Morrison, Esther J. Rios-Lopez, Shirly Guedez-Gonzales, John P. Grieco, Kirk Mundal, Theeraphap Chareonviriyaphap, and Nicole L. Achee. 2011. Initial Assessment of the Acceptability of a Push-Pull Aedes aegypti Control Strategy in Iquitos, Peru and Kanchanaburi, Thailand. The American Journal of Tropical Medicine and Hygiene 84 (2): 208-217. https://doi.org/10.4 269/ajtmh.2011.09-0615.

Pereira, T., D Roiz, R. Lourenco do Oliveira, and C. Paupy. 2020. A Systematic Review: Is Aedes albopictus an Efficient Bridge Vector for Zoonotic Arboviruses? Pathogens (Basel, Switzerland). Pathogens, July 4. https://doi.org/10.3390/pathogens9040266.

Perez-Guerra, C.L., E. Zielinski-Gutierrez, D. Vargas-Torres, and G.G. Clark. 2009. Community Beliefs and Practices about Dengue in Puerto Rico. Revista Panamericana de Salud Publica 25 (March): 218-226.

Powell, J.R., Gloria-Soria A., Kotsakiozi P. and Ding-Guo Gao. 2000. Recent History of Aedes aegypti: Vector Genomics and Epidemiology Records. Bioscience 68(11), 854860. https://doi.org/10.1093/biosci/biy119

Qsim, Muhammad, Usman Ali Ashfaq, Muhammad Zubair Yousaf, Muhammad Shareef Masoud, Ijaz Rasul, Namrah Noor, and Azfar Hussain. 2017. Genetically Modified Aedes aegypti to Control Dengue: A Review. Critical Reviews in Eukaryotic 
Gene Expression 27 (4): 331-340. https://doi.org/10.1615/CritRevEukaryotGeneExpr 2017019937.

Revay, Edita E., Gunter C. Müller, Whitney A. Qualls, Daniel Kline, Diana P. Naranjo, Kristopher L. Arheart, Vasiliy D. Kravchenko, et al. 2014. Control of Aedes albopictus with Attractive Toxic Sugar Baits (ATSB) and Potential Impact on Non-Target Organisms in St. Augustine, Florida. Parasitology Research 113 (1): 73-79. https://doi .org/10.1007/s00436-013-3628-4.

Roiz, David, Anne L. Wilson, Thomas W. Scott, Dina M. Fonseca, Frédéric Jourdain, Pie Müller, Raman Velayudhan, and Vincent Corbel. 2018. Integrated Aedes Management for the Control of Aedes-Borne Diseases. PLOS Neglected Tropical Diseases 12 (12): e0006845. https://doi.org/10.1371/journal.pntd.0006845.

Ryan, Peter A., Andrew P. Turley, Geoff Wilson, Tim P. Hurst, Kate Retzki, Jack Brown-Kenyon, Lauren Hodgson, et al. 2019. Establishment of WMel Wolbachia in Aedes aegypti Mosquitoes and Reduction of Local Dengue Transmission in Cairns and Surrounding Locations in Northern Queensland, Australia. Gates Open Research 3: 1547. https://doi.org/10.12688/gatesopenres.13061.2.

Samuel, Moody, Dorit Maoz, Pablo Manrique, Tara Ward, Silvia Runge-Ranzinger, Joao Toledo, Ross Boyce, and Olaf Horstick. 2017. Community Effectiveness of Indoor Spraying as a Dengue Vector Control Method: A Systematic Review. PLoS Neglected Tropical Diseases 11 (8): e0005837. https://doi.org/10.1371/journal.pntd.000 5837.

Scott, Jeffrey G., Melissa Hardstone Yoshimizu, and Shinji Kasai. 2015. Pyrethroid Resistance in Culex pipiens Mosquitoes. Pesticide Biochemistry and Physiology 120 (May): 68-76. https://doi.org/10.1016/j.pestbp.2014.12.018.

Sherwood, J.A., S.V. Stehman, J.J. Howard, and J. Oliver. 2020. Cases of Eastern Equine Encephalitis in Humans Associated with Aedes canadensis, Coquillettidia perturbans and Culiseta melanura Mosquitoes with the Virus in New York State from 1971 to 2012 by Analysis of Aggregated Published Data. Epidemiology \& Infection 148. https://doi .org/10.1017/S0950268820000308.

Soper, Fred L. 1963. The Elimination of Urban Yellow Fever in the Americas Through the Eradication of Aedes aegypti. American Journal of Public Health and the Nation's Health 53 (1): 7-16.

Sousa, L., Mello De, D. Cedrim, A. Garcia, P. Missier, U. Anderson, O. Anderson, and A. Romanovsky. 2017. VazaDengue: An Information System for Preventing and Combating Mosquito-Borne Diseases with Social Networks. School of Computing Science Technical Report Series. https://eprint.ncl.ac.uk/240257.

Sulistyawati, Sulistyawati, Fardhiasih Dwi Astuti, Sitti Rahmah Umniyati, Tri Baskoro Tunggul Satoto, Lutfan Lazuardi, Maria Nilsson, Joacim Rocklov, Camilla Andersson, and Åsa Holmner. 2019. Dengue Vector Control through Community Empowerment: Lessons Learned from a Community-Based Study in Yogyakarta, Indonesia. International Journal of Environmental Research and Public Health 16 (6). https://doi.org/10.3390/ijerph16061013.

Thomas, D.D., C.A. Donnelly, R.J. Wood, and L.S. Alphey. 2000. Insect Population Control Using a Dominant, Repressible, Lethal Genetic System. Science (New York, N.Y.) 287 (5462): 2474-2476. https://doi.org/10.1126/science.287.5462.2474.

UNICEF/UNDP/World Bank/WHO. 2017. Global Vector Control Response 20172030. Special Programme for Research and Training in Tropical. https://apps.who .int/iris/handle/10665/259002.

Vilibic-Cavlek, Tatjana, Vladimir Savic, Tamas Petrovic, Ivan Toplak, Ljubo Barbic, Dusan Petric, Irena Tabain, et al. 2019. Emerging Trends in the Epidemiology of West 
Nile and Usutu Virus Infections in Southern Europe. Frontiers in Veterinary Science 6: 437. https://doi.org/10.3389/fvets.2019.00437.

Weaver, Scott C., and William K. Reisen. 2010. Present and Future Arboviral Threats. Antiviral Research 85 (2): 328-345. https://doi.org/10.1016/j.antiviral.2009.10.008.

Werth, Julia. 2019. As Massachusetts and Rhode Island Begin Aerial Spraying, Mosquitoes in Southeast Connecticut Test Positive for Eastern Equine Encephalitis. The Connecticut Examiner (blog). September 10. https://ctexaminer.com/2019/09/10 /as-massachusetts-and-rhode-island-begin-aerial-spraying-mosquitoes-in-southeast -connecticut-test-positive-for-eastern-equine-encephalitis/.

WHOPES. 2010. WHO Pesticide Evaluation Scheme: 50 Years of Global Leadership. WHO/ HTM/NTD/WHOPES/2010.2. Geneva: WHO. http://www.who.int/whopes/reso urces/978924159927/en/.

Wilder-Smith, Annelies, Duane J. Gubler, Scott C. Weaver, Thomas P. Monath, David L. Heymann, and Thomas W. Scott. 2017. Epidemic Arboviral Diseases: Priorities for Research and Public Health. The Lancet Infectious Diseases 17 (3): e101-106. https://do i.org/10.1016/S1473-3099(16)30518-7.

Wilson, Anne L., Marleen Boelaert, Immo Kleinschmidt, Margaret Pinder, Thomas W. Scott, Lucy S. Tusting, and Steve W. Lindsay. 2015. Evidence-Based Vector Control? Improving the Quality of Vector Control Trials. Trends in Parasitology 31 (8): 380-390. https://doi.org/10.1016/j.pt.2015.04.015.

World Health Organization. 1997. Dengue Haemorrhagic Fever: Diagnosis, Treatment, Prevention and Control. World Health Organization. https://apps.who.int/iris/handle /10665/41988.

World Health Organization. 2012. Global Strategy for Dengue Prevention and Control, 20122020. Geneva: World Health Organization. http://apps.who.int/iris/bitstream/ 10665/75303/1/9789241504034_eng.pdf.

World Health Organization. 2020. WHO. World Health Organization. http://www .who.int/neglected_diseases/vector_ecology/mosquito-borne-diseases/en/.

Zanotti, Gabriela, María Sol De Majo, Iris Alem, Nicolás Schweigmann, Raúl E Campos, and Sylvia Fischer. 2015. New Records of Aedes aegypti at the Southern Limit of Its Distribution in Buenos Aires Province, Argentina. Journal of Vector Ecology 40 (2): 408-411. 